\title{
Astragalus membranaceus and Salvia miltiorrhiza Ameliorate Lipopolysaccharide-Induced Acute Lung Injury in Rats by Regulating the Toll-Like Receptor 4/Nuclear Factor-Kappa B Signaling Pathway
}

\author{
Li Qin (D, ${ }^{1}$ Hong-Ling Tan, ${ }^{2}$ Yu-Guo Wang, ${ }^{1}$ Cheng-Yong Xu, ${ }^{1}$ Jian Feng, \\ Min Li ${ }^{1},^{1}$ and Yong-Qi Dou ${ }^{1}{ }^{1}$ \\ ${ }^{1}$ Department of Traditional Chinese Medicine, Chinese PLA General Hospital, Beijing 100853, China \\ ${ }^{2}$ Institute of Radiation Medicine Sciences, Academy of Military Medical Sciences, Beijing 100850, China \\ Correspondence should be addressed to Min Li; kittenlee1115@126.com and Yong-Qi Dou; dyqi_301@yeah.net
}

Received 10 January 2017; Revised 26 April 2017; Accepted 20 July 2017; Published 29 January 2018

Academic Editor: Ling Yang

Copyright (C) $2018 \mathrm{Li}$ Qin et al. This is an open access article distributed under the Creative Commons Attribution License, which permits unrestricted use, distribution, and reproduction in any medium, provided the original work is properly cited.

\begin{abstract}
Astragalus membranaceus and Salvia miltiorrhiza (AM/SM) are well used in Traditional Chinese Medicines (TCM) for nourishing Qi and activating blood circulation method. From TCM theory, the pathogenesis of acute lung injury (ALI) was determined as Qi deficiency and blood stagnation. In this study, we are aiming to investigate the protective and therapeutic effects of AM/SM on a rat model of lipopolysaccharide- (LPS-) induced ALI in rats and to elucidate potential molecular mechanisms. ALI was induced by intratracheal instillation of LPS $(5 \mathrm{mg} / \mathrm{kg})$ in Sprague-Dawley rats. SM/AM was given orally before and after LPS administration. Results demonstrated that AM/SM attenuated lung histopathological changes induced by LPS, decreased wet/dry weight ratios and protein concentrations, and inhibited the production of tumor necrosis factor-alpha (TNF- $\alpha$ ) and interleukin- 6 (IL-6) in BALF. Moreover, AM/SM significantly downregulated protein and mRNA expression of toll-like receptors 4 (TLR-4), interleukin-1 receptor-associated kinase-1 (IRAK-1), and nuclear factor-kappa B (NF- $\kappa$ B/p65). These findings suggest that AM/SM showed protective and therapeutic effects in LPS-induced ALI rat through modulating TLR-4 signaling pathways. Nourishing Qi and activating blood circulation may be a beneficial treatment for ALI.
\end{abstract}

\section{Introduction}

Acute lung injury (ALI) and its more severe form, acute respiratory distress syndrome, are characterized by uncontrolled hyperinflammatory responses in the lung airspaces and lung parenchyma and involves alveolar capillary membrane damage, increased vascular permeability, neutrophils recruitment, pulmonary edema, and respiratory failure [1-3]. The mortality rate for acute respiratory distress syndrome has remained at approximately $40 \%$ over the past decades despite major advances in antimicrobial therapy and supportive care [4], and its cause has direct injury, such as pneumonia or aspiration, and indirect injury, such as sepsis or trauma [5]. As the primary component of the outer membrane of Gram-negative bacteria, lipopolysaccharide (LPS), is an important risk factor for acute inflammatory responses and overwhelming innate immunity in ALI [6]. Intratracheal administration of LPS is a widely acceptable method to induce a clinical relevant model of ALI and has been widely used to study the pathogenesis of ALI and potential therapies [7].

The toll-like receptor superfamily (TLRs), a major connective factor between innate and adaptive mucosal immune responses [8], can recognize pathogen-associated molecular patterns (PAMPs), such as TLR-4 recognition of LPS. TLR-4 is a transmembrane protein that acts as a signal transduction molecule [9]. Once stimulated by LPS, TLR4 is activated and recruits the adaptor molecule myeloid differentiation primary response gene 88 (MyD88) to the cytoplasmic domain of the receptor, which subsequently 
induces the association and phosphorylation of interleukin1 receptor- (IL-1R-) associated protein kinase 1 (IRAK-1) [10, 11 ], ultimately promoting the activation of the transcription factor-nuclear factor-kappa B (NF- $\kappa \mathrm{B})[12]$. Activated NF- $\kappa \mathrm{B}$ initiates the excess production of proinflammatory cytokines such as tumor necrosis factor-alpha (TNF- $\alpha$ ), interleukin(IL-) 6 , and IL-1 $\beta[13,14]$, resulting in an uncontrolled inflammatory response and ALI.

Astragalus membranaceus Bunge (of the Leguminosae family) is well used in Traditional Chinese Medicines (TCM) for the treatment of general weakness to increase overall vitality according to the Chinese Pharmacopoeia (2015). This herb has been extensively studied, mainly for its three main groups of biologically active compounds (polysaccharides, flavonoids, and saponins) [15], and has been shown to exhibit immunomodulating, anti-inflammatory, antioxidant, and antiviral activities in modern medicine [16]. Salvia miltiorrhiza Bunge (of the Lamiaceae family) is highly valued in TCM for promoting blood circulation to remove blood stasis according to Chinese Pharmacopoeia (2015). The principal bioactive components in this herb are hydrophilic phenolic acids and tanshinones [17]. Salvia miltiorrhiza possesses significant antioxidant, anti-inflammatory, and antineoplastic activities and has been used in various diseases, especially in coronary heart disease and cerebrovascular disease [18-21]. Astragalus membranaceus and Salvia miltiorrhiza (AM/SM) constitute nourishing Qi and activating blood circulation herbs to prevent and treat LPS-induced ALI in rats. Our previous clinical studies have found that intervention with supplementing Qi and activating blood circulation herbs can effectively protect the lung function in ALI patients who received ventilation [22]. Following from these studies, we aimed to further elucidate the potential effective mechanisms at the molecular level. Because of the important role of the TLR-4 signaling pathway in inflammation, we tested whether AM/SM could ameliorate LPS-induced ALI through regulation of TLR-4, IRAK-1, and NF- $\kappa \mathrm{B} / \mathrm{p} 65$ in this study, hypothesizing that it could explain the possible role of $\mathrm{AM} / \mathrm{SM}$ in the protection of inflammatory response-induced lung injury.

\section{Material and Methods}

2.1. Animals. Male Sprague-Dawley rats weighing 200-250 g were provided by the Animal Raising Center of the Academy of Military Medical Science [animal certificate number: SCXK (Army)-2012-0004]. Rats were housed on a $12 \mathrm{~h}$ light/dark cycle in a temperature- and humidity-controlled room and maintained on a standard diet and water ad libitum. Animal protocols were approved by the Ethics Committee of the Academy of Military Medical Science (Beijing, China). Experimental studies were performed in accordance with the National Institutes of Health Guide for the Care and Use of Laboratory Animals. All surgery was carried out under sodium pentobarbital anesthesia.

2.2. Chemicals and Reagents. LPS (Escherichia coli 055:B5) was purchased from Sigma-Aldrich (St. Louis, MO, USA). Dexamethasone (Dex) acetate tablets (number H12020122) were purchased from Tianjin Lisheng Pharmaceutical Co., Ltd. (Tianjin, China). Bicinchoninic acid (BCA) assay kits were provided by Applygen Technologies Inc. (Beijing, China). Rat TNF- $\alpha$ and IL- 6 enzyme-linked immunosorbent assay (ELISA) kits were obtained from American R\&D Corp. (Minneapolis, MN, USA). Rabbit anti-TLR-4, anti-IRAK-1, and anti-NF- $\kappa \mathrm{B} / \mathrm{p} 65$ antibodies were obtained from Bioss Biotechnology Co., Ltd. (Beijing, China). RNApure kits, MMLV III First-Strand cDNA synthesis kits, and Green qPCR MasterMix were purchased from Biomed Gene Technology Co., Ltd. (Beijing, China).

2.3. Plant Materials and Preparation. AM/SM was prepared and provided by the Traditional Chinese Medicine dispensary at the General Hospital of the Chinese People's Liberation Army, Beijing, China. AM and SM were mixed at a ratio of $2: 1(\mathrm{w} / \mathrm{w})$, decocted, filtered, and concentrated at the Institute of Radiation Medicine, Academy of Military Medical Science. For the traditional decoction, the above mixed dry herbs were soaked into 10 times of water for $1 \mathrm{~h}$ and boiled $30 \mathrm{~min}$ to get the extracted solution and then boiled for another $30 \mathrm{~min}$ to get the extracted solution; this preparation method was established according to the ancient Chinese method. The two times extracted solutions were mixed together and concentrated on a rotary vacuum evaporator. The final concentration was each millilitre containing $0.59 \mathrm{~g}$ of crude drugs, which was in accordance with the dose calculation of the body surface area ratio between humans and rats.

2.4. Construction of ALI Model and AM/SM Treatment. After a 1-week acclimatization period, forty rats were randomly divided into five groups of eight per group: sham group, LPS group, Dex (5 mg/kg) group, AM/SM treatment group (herb I), and AM/SM prophylaxis therapeutic group (herb II). Rats were either given saline or drugs. The herb II group was given intragastric administration of AM/SM $(0.59 \mathrm{~g} / \mathrm{mL}, 10 \mathrm{~mL} / \mathrm{kg})$ once per day for 3 consecutive days. Rats in the sham, LPS, Dex, and herb I groups received normal saline at a dose of $10 \mathrm{~mL} / \mathrm{kg}$ once per day for 3 consecutive days.

On day $3,2 \mathrm{~h}$ after administration, rats were anesthetized with an intraperitoneal injection of pentobarbital sodium $(30 \mathrm{mg} / \mathrm{kg})$ solution $(1 \mathrm{~mL} / \mathrm{kg}$ of $3 \%$ pentobarbital sodium solution). Skin preparation was performed at the throat of the rats. After regular disinfection, the throat was incised longitudinally with the rat head in the higher position. Skin and subcutaneous tissue were separated layer by layer, until the trachea was exposed. Then, rats for the model of ALI (LPS, Dex, and herbs groups) received a single intratracheal instillation with $5 \mathrm{mg} / \mathrm{kg}$ LPS $(2.5 \mathrm{mg} / \mathrm{mL}$, diluted with phosphatebuffered saline (PBS), $0.2 \mathrm{~mL} / 100 \mathrm{~g}$ body weight) to induce ALI. Control rats from sham group were given intratracheal administration of an equal volume of sterile PBS in the same way. Finally, the incision was sutured.

Two hours after the intratracheal administration, herb I and II groups received AM/SM $(0.59 \mathrm{~g} / \mathrm{mL}, 10 \mathrm{~mL} / \mathrm{kg})$, the Dex group received dexamethasone $(5 \mathrm{mg} / \mathrm{kg})$, and the sham 
and LPS groups received normal saline $(1 \mathrm{~mL} / \mathrm{kg})$ once per day for 3 consecutive days. Drug doses were determined based on previous studies and our preliminary experiments.

\subsection{Collection of Bronchoalveolar Lavage Fluid and Lung} Tissues. Six hours after the last administration, rats were killed by exsanguination and the thoracic cavity was surgically exposed. The hilum of the right lung was ligated and the left lung was lavaged with $2 \mathrm{~mL}$ ice-cold PBS ( $\mathrm{pH} 7.2$ ) three times (total volume $6 \mathrm{~mL}$ ). The recovery ratio of the fluid was about $90 \%$. Collected bronchoalveolar lavage fluid (BALF) was immediately centrifuged at $1,500 \mathrm{rpm}$ for $10 \mathrm{~min}$ at $4^{\circ} \mathrm{C}$ and the supernatant stored at $-80^{\circ} \mathrm{C}$ for inflammatory cytokine analysis. The bilateral lungs were dissected and left lungs were snap frozen in liquid nitrogen and stored at $-80^{\circ} \mathrm{C}$ for quantitative real-time PCR (qPCR).

2.6. Measurement of Lung Wet/Dry Ratio and Protein Concentration in BALF. The wet/dry ratio is an index of pulmonary edema. The inferior lobe of the right lung was excised and the wet weight was recorded before being placed in an incubator at $60^{\circ} \mathrm{C}$ for $72 \mathrm{~h}$ to obtain the dry weight. The lung wet/dry weight ratio was calculated for each group. To evaluate vascular permeability in the lung, the total protein concentration in BALF was determined using the BCA method according to the manufacturer's instructions.

2.7. ELISA Assay for TNF- $\alpha$ and IL-6 in BALF. Levels of TNF$\alpha$ and IL- 6 in BALF were determined by ELISA according to the manufacturer's instructions. The optical density of each well was measured at $450 \mathrm{~nm}$ using a microplate spectrophotometer, and results were calculated according to standard curves.

2.8. Histological Examination. The middle lobe of the right lung was excised, fixed in $4 \%$ paraformaldehyde for $24 \mathrm{~h}$ at $4^{\circ} \mathrm{C}$, embedded in paraffin, and sectioned ( $4 \mathrm{~mm}$ thick). Hematoxylin and eosin ( $\mathrm{H} \& \mathrm{E})$ staining was performed according to the standard protocol. Pathological changes in lung tissue were observed under a light microscope.

2.9. Immunohistochemistry. The upper lobe of the right lung was excised and fixed in $4 \%$ paraformaldehyde for $24 \mathrm{~h}$ at $4^{\circ} \mathrm{C}$, embedded in paraffin, sectioned (4-mm thick), deparaffinized with xylene, and hydrated with a graded ethanol series. Sections were then heated in citrate buffer (pH 6.0) in a microwave. Endogenous peroxidase activity was blocked with 3\% H2O2 for $10 \mathrm{~min}$. Sections were washed with PBS and blocked with $10 \%$ goat serum for $15 \mathrm{~min}$ at room temperature. Sections were then washed with PBS again and incubated with a rabbit anti-TLR-4 antibody (1:200 dilution), a rabbit anti-IRAK-1 antibody (1:400 dilution), and a rabbit anti-NF- $\kappa \mathrm{B} / \mathrm{p} 65$ antibody (1:200 dilution), respectively, overnight at $4^{\circ} \mathrm{C}$. Negative controls were incubated in PBS in place of the specific primary antibody. The following day, after washing, sections were incubated with biotinylated secondary antibodies conjugated to goat antirabbit antibody at room temperature for $20 \mathrm{~min}$ and then with streptavidin-horseradish peroxidase. Finally, all sections were stained with $3,3^{\prime}$-diaminobenzidine tetrahydrochloride and lightly counterstained with hematoxylin. The expression and localization of TLR -4 and NF- $\kappa$ B protein were examined under a light microscope. Six visual fields were randomly selected from each section for quantitative analyses. The integrated optical density (IOD) of the positive products in the lung was measured by 6.0 analysis system.

2.10. RNA Extraction and $q P C R$. qPCR was used to measure transcript levels of TLR-4, IRAK-1, and NF- $\kappa$ B. Total RNA was extracted from $50 \mathrm{mg}$ of lung tissue using a Biomed RNApure kit. One microgram of RNA was reverse transcribed into cDNA using the M-MLV III First-Strand Synthesis System. The system was performed at $42^{\circ} \mathrm{C}$ for $50 \mathrm{~min}$ and terminated by deactivation of the enzyme at $85^{\circ} \mathrm{C}$ for $15 \mathrm{~min}$. In a parallel experiment, glyceraldehyde 3-phosphate dehydrogenase (GAPDH) was used as an internal control. PCR primers were TLR-4 (140 bp): 5'-AGTTGGCTCTGCCAAGTCTCAGAT$3^{\prime}$ (forward), 5' -TGGCACTCATCAGGATGACACCAT$3^{\prime}$ (reverse); NF- $\kappa \mathrm{B}$ (145 bp): $5^{\prime}$-ACATCCCTCAGCACCATCAA-3 $3^{\prime}$ (forward), $5^{\prime}$-TTGGTACCATGGCTGAGGAG$3^{\prime}$ (reverse); and GAPDH (116 bp): $5^{\prime}$-AAGGGCTCATGACCACAGTC-3' (forward), 5'-GGATGCAGGGATGATGTTCT-3' (reverse). qPCR reaction conditions were performed using SYBR Green qPCR MasterMix and a StepOne ${ }^{\mathrm{TM}}$ Real-Time PCR System (StepOne 7900; Applied Biosystems, Carlsbad, CA, USA). PCR amplification conditions were initial denaturation at $95^{\circ} \mathrm{C}$ for $10 \mathrm{~min}$, followed by 40 cycles of denaturing at $95^{\circ} \mathrm{C}$ for $15 \mathrm{~s}$, annealing at $60^{\circ} \mathrm{C}$ for $1 \mathrm{~min}$, and a final extension step at $60^{\circ} \mathrm{C}$ for $5 \mathrm{~min}$. We used a comparative method $\left(2^{-\Delta \Delta \mathrm{CT}}\right)$ to calculate the amount of mRNA of the target gene, where $\Delta \mathrm{Ct}=\mathrm{Ct}$ (target gene) $-\mathrm{Ct}$ (reference gene) and $\Delta \Delta \mathrm{Ct}=\Delta \mathrm{Ct}$ (sample) $-\Delta \mathrm{Ct}$ (control).

2.11. Statistical Analysis. Data were analyzed using SPSS version 17.0 statistical software (SPSS, Chicago, IL, USA). Values are expressed as mean \pm standard deviation (SD). One-way analysis of variance (ANOVA) was used for multiple comparisons among groups. A $P$-value $<0.05$ was considered statistically significant.

\section{Results}

3.1. Effect of AM/SM on Lung Wet/Dry Ratio and Protein Concentration in BALF. Lung wet/dry ratios and BALF protein concentrations significantly increased after LPS challenge compared with the sham group $(P<0.01)$ (Figures 1 and 2 ). However, the Dex and herb groups showed obvious decreases in ratios and protein concentrations compared with the LPS group $(P<0.01)$, and the Dex and herb II groups were significantly lower than the herb I group $(P<0.05$ and $P<$ 0.01 , resp.). No significant differences in wet/dry ratios were found between the Dex group and the herb II group. Results suggested that SM/AM herbs could alleviate LPS-induced 


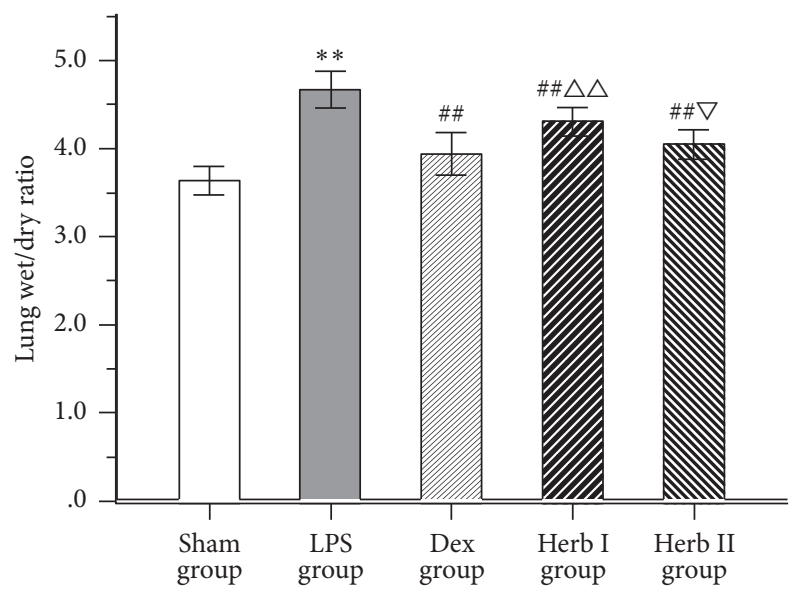

FIGURE 1: Effect of AM/SM on the lung wet/dry ratio. Values were expressed as mean $\pm \mathrm{SD}(n=10) .{ }^{* *} P<0.01$ versus sham group. ${ }^{\# \#} P<0.01$ versus LPS group. ${ }^{\triangle \triangle} P<0.01$ versus Dex group. ${ }^{\nabla} P<$ 0.05 versus group $\mathrm{I}$.

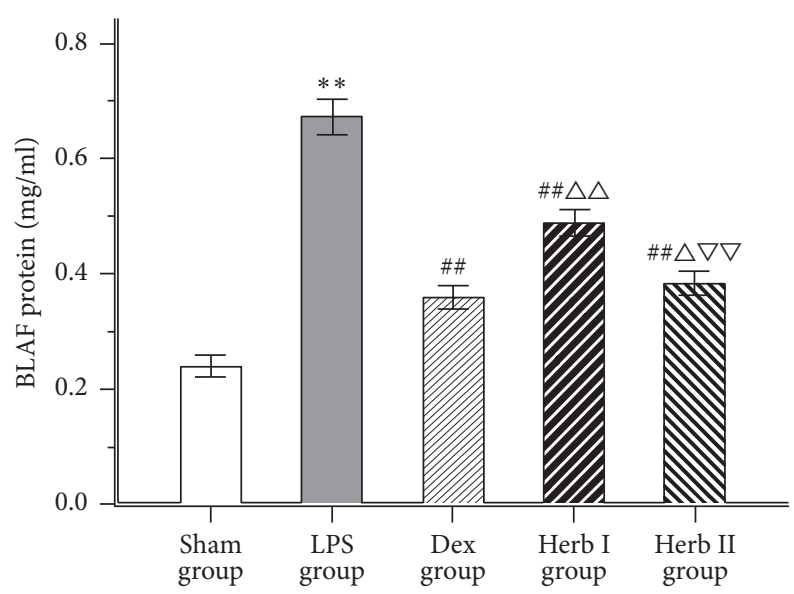

FIGURE 2: Effect of AM/SM on the total protein concentration in BLAF. Values were expressed as mean $\pm \mathrm{SD}(n=10) .{ }^{* *} P<0.01$ versus sham group. ${ }^{\# \#} P<0.01$ versus LPS group. ${ }^{\triangle} P<0.05$ and ${ }^{\triangle} \triangle P<0.01$ versus Dex group. ${ }^{\nabla} P<0.01$ versus group I.

pulmonary edema and decrease vascular permeability in the lung, exploiting preventive and therapeutic effects.

\subsection{Effect of AM/SM on Lung Histology. Histopathological} analyses were performed to investigate the effects of AM/SM on physiological parameters. Lung tissue in the sham group showed a normal structure, with no evident histopathological changes. In contrast, lung tissue from the LPS group showed significant pathological changes, with pulmonary congestion, interstitial edema, alveolar wall thickness, and mass inflammatory cell infiltration. The pathological changes in lung tissue were alleviated with AM/SM and dexamethasone treatment compared with the LPS group. The results suggested that AM/SM could attenuate the severity of LPS-induced lung injury and improve the condition of the lung tissue (Figure 3).
3.3. Effect of AM/SM on TNF- $\alpha$ and IL-6 Levels in BALF. To determine the effects of AM/SM on LPS-induced cytokine production, concentrations of the proinflammatory cytokines TNF- $\alpha$ and IL- 6 in BALF were measured using ELISA. LPS challenge significantly increased TNF- $\alpha$ and IL6 levels in BALF compared with the control group (Figures 4(a) and 4(b)). Dexamethasone and herb treatment decreased TNF- $\alpha$ and IL- 6 levels compared with the LPS group. There were no significant differences in TNF- $\alpha$ levels in BALF between the Dex and herb II groups. The herb II group showed significantly reduced TNF- $\alpha$ and IL- 6 levels compared with the herb I group. The results suggest that AM/SM could attenuate the inflammatory reaction in LPS-induced ALI.

3.4. Effect of AM/SM on the Protein Expression of TLR-4, $I R A K-1$, and $N F-\kappa B$. To further investigate the mechanisms of action of AM/SM on LPS-induced ALI, mRNA and protein levels of TLR-4, IRAK-1, and NF- $\kappa \mathrm{B} / \mathrm{p} 65$ were detected using qPCR and immunohistochemistry, respectively. As shown in Figure 5(a), a few positive products (the expression of TLR-4 protein, IRAK-1 protein, and NF- $\kappa$ B protein) were observed in lung tissue of the sham group. In contrast, lung tissue from the LPS group showed a large number of positive products. Compared with the LPS group, positive products in lung tissue were reduced with AM/SM and dexamethasone treatment. TLR-4 protein and IRAK-1 protein in the herb II group were lower than that in the herb I group. No significant differences in TLR-4 and IRAK-1 protein were found between the Dex group and the herb II group (Figures 5(b), 5(c), and $5(d))$.

3.5. Effect of $A M / S M$ on the mRNA Expression of TLR-4, IRAK-1, and $N F-\kappa B$. As for the PCR results, expression of TLR-4 mRNA, IRAK-1 mRNA, and NF- $\kappa$ B/p65 mRNA was markedly upregulated compared with the sham group with LPS challenge, while treatment with AM/SM and dexamethasone significantly decreased the levels compared with the LPS group. TLR-4, IRAK-1, and NF- $\kappa \mathrm{B} / \mathrm{p} 65$ mRNA levels in the herb II group were lower than that in the herb I group. No significant differences in TLR-4 and IRAK-1 mRNA were found between the Dex group and the herb II group (Figures 6(b), 6(c), and 6(d)).

\section{Discussion}

The pathological characteristics of ALI are alveolar capillary permeability increases, extensive neutrophils infiltration, inflammatory mediators release, and pulmonary edema [23]. Inflammatory cell recruitment is an important initial step in the host defense against inhaled toxicants, leading to the pulmonary capillary damage and an increase in alveolar epithelial permeability [24]. Then a large number of inflammatory mediators are released, resulting in inflammation. Diffuse alveolar damage occurs with the cascading process of inflammation, developed from epithelial barrier dysfunction, 

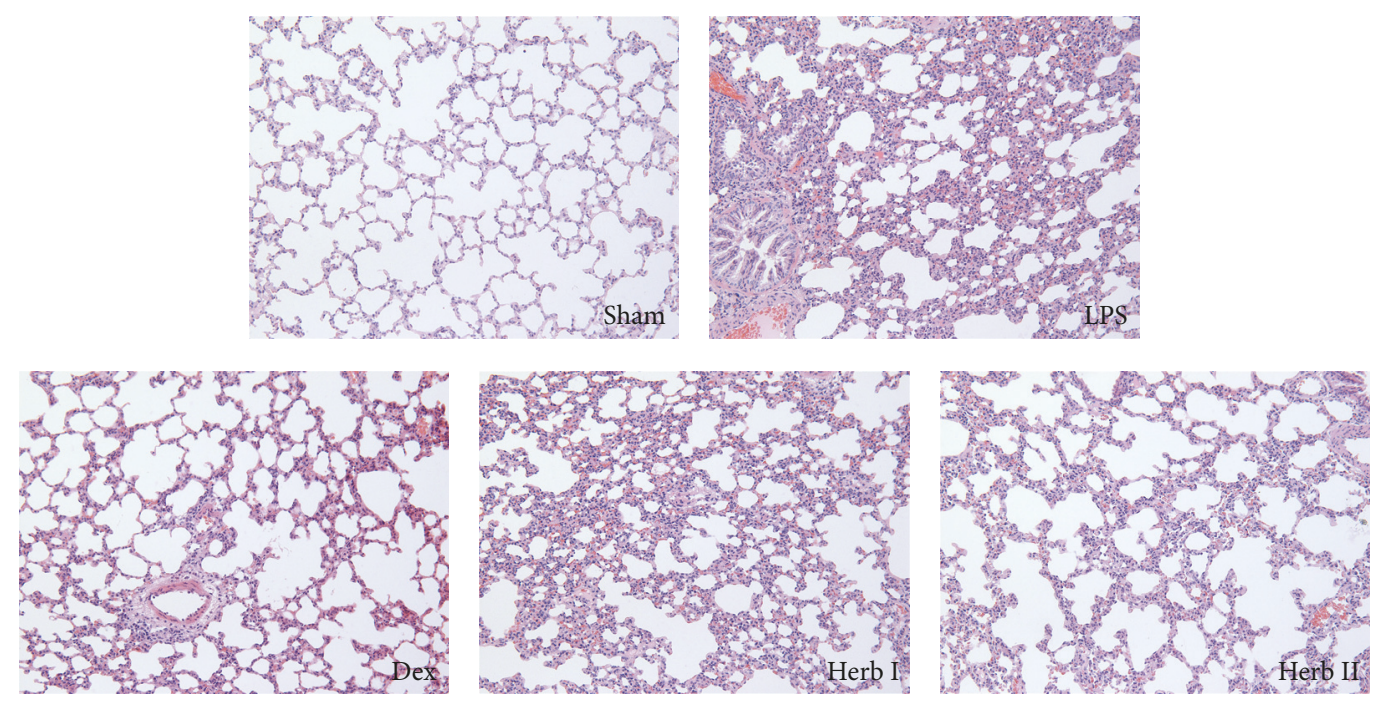

FIGURE 3: Effect of AM/SM on lung histological examination (H\&E-stain, magnification $\times 200)$. Sham group exhibiting a normal structure of lung tissue; LPS group showing significant pathological changes, with pulmonary congestion, interstitial edema, alveolar wall thickness, and mass inflammatory cell infiltration; Dex group, herb I group, and herb II group showing a lower inflammatory cell infiltration and thickening of the alveolar wall compared to LPS group.

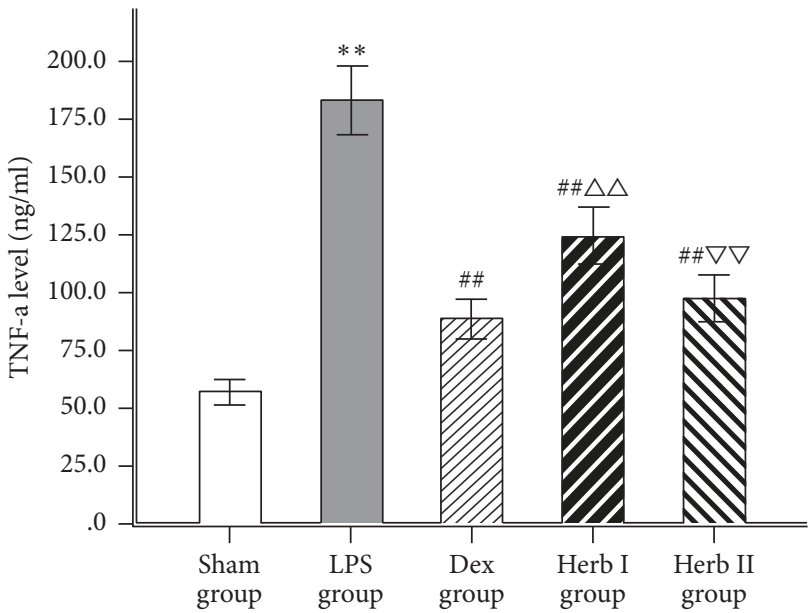

(a)

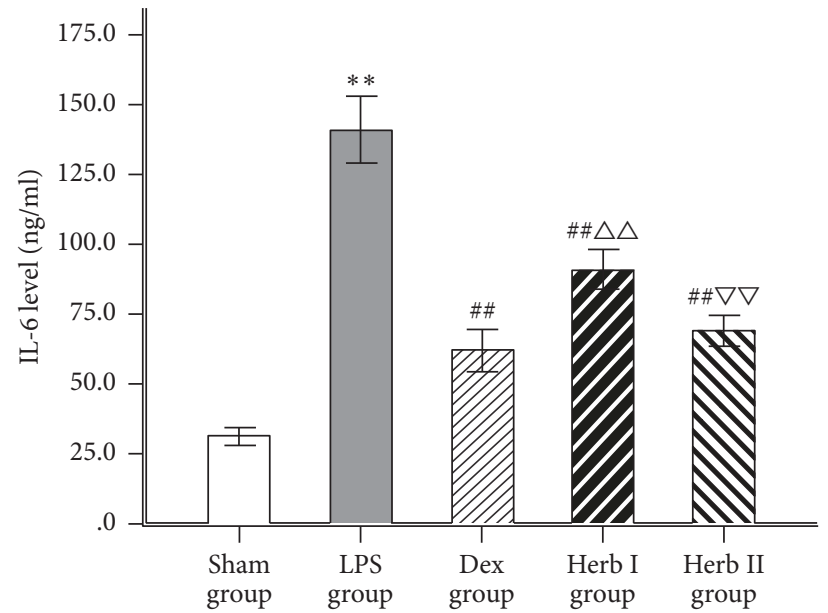

(b)

Figure 4: Effect of AM/SM on the content of TNF- $\alpha$ (a) and IL-6 (b) in BALF (pg/ml). Values were expressed as mean \pm SD $(n=10)$. ${ }^{* *} P<0.01$ versus sham group. ${ }^{\# \#} P<0.01$ versus LPS group. ${ }^{\triangle \triangle} P<0.01$ versus Dex group. ${ }^{\nabla \nabla} P<0.01$ versus group I.

endothelial dysfunction, and pulmonary edema [25]. In LPSinduced ALI models via intratracheal instillation, LPS triggers the TLR-4 signaling pathway and induces the expression of inflammatory cytokines, resulting in loss of microvascular and epithelial integrity and increased interstitial and alveolar edema $[7,26]$.

TCM formulae generally claim efficacy in treating lung disease based on its multiple bioactive compounds and good compliance for long-term use [27]. Ethnological medicinal plants have gained increased attention as they are an important source of complementary and alternative medicine [28]. From TCM theory and our own previous studies [22], the pathogenesis of ALI was determined as Qi deficiency and blood stagnation; therefore, we used AM/SM herbs to nourish Qi and activate blood circulation in this study. We investigated the protective and therapeutic effects of AM/SM on a rat model of LPS-induced ALI to elucidate potential molecular mechanisms. We found that AM/SM attenuated LPS-induced lung histopathological changes, decreased the wet/dry weight ratio, and inhibited or suppressed protein and proinflammatory cytokine concentrations in BALF. In particular, AM/SM significantly downregulated protein and mRNA expression of TLR-4, IRAK-1, and NF- $\kappa$ B. These results suggest that the effects of AM/SM on LPS-induced ALI are possibly because of its ability to modulate TLR- 4 signaling pathways and that this particular method for nourishing Qi 


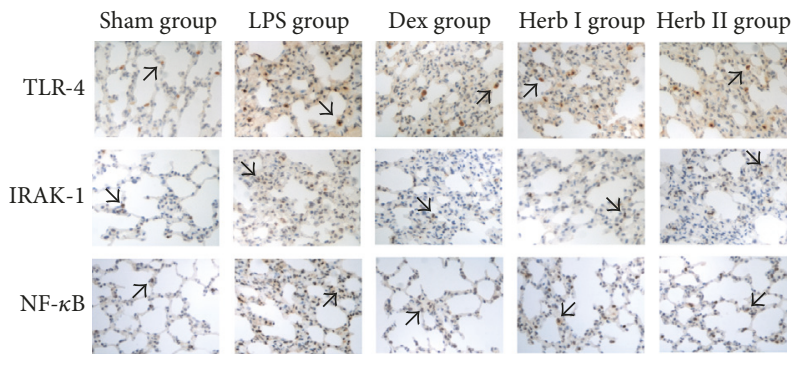

(a)

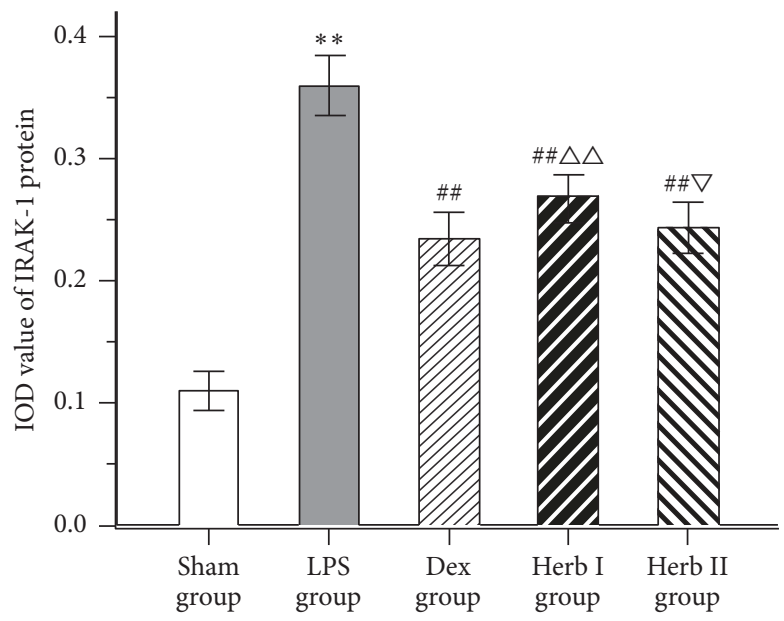

(c)

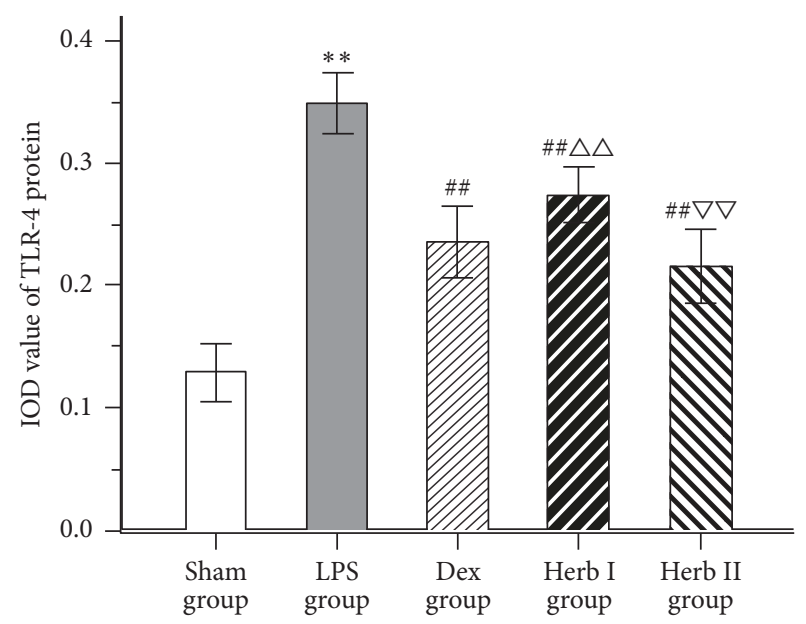

(b)

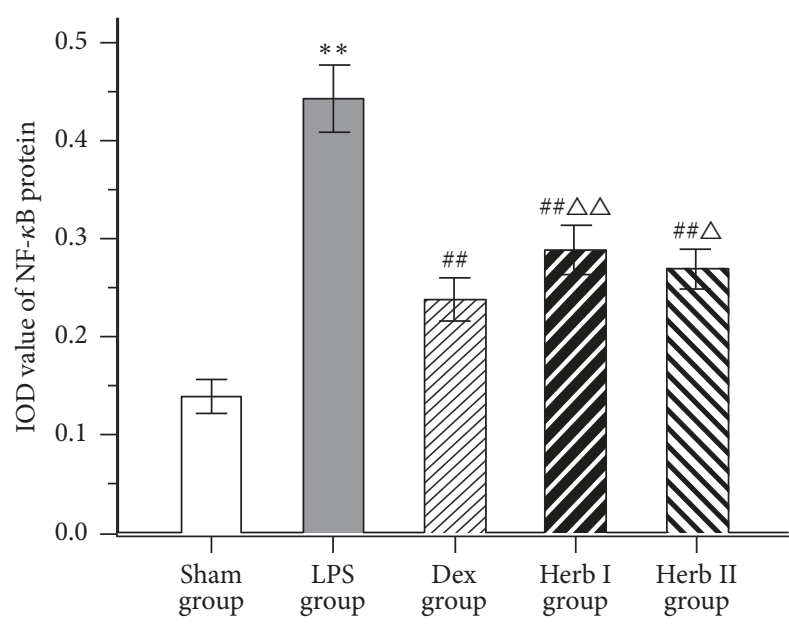

(d)

FIGURE 5: Effects of AM/SM on the TLR-4, IRAK-1 and NF- $\kappa$ B protein expression of lung tissues. (Immunohistochemistry-stain, magnification $\times 400$ ). (a) Lung sections from sham group, LPS group, Dex group, herb I group, and herb II group were stained with TLR-4, IRAK-1, and NF- $\kappa$ B antibody and hematoxylin. Positive products were represented by a brown-yellow color (black Arrow). (b) IOD value of TLR-4 protein expression; (c) IOD value of IRAK-1 protein expression; (d) IOD value of NF- $\kappa$ B protein expression. Values were expressed as mean \pm SD $(n=10) .{ }^{* *} P<0.01$ versus sham group. ${ }^{\# \#} P<0.01$ versus LPS group. ${ }^{\triangle} P<0.05$ and ${ }^{\triangle} P<0.01$ versus Dex group. ${ }^{\nabla} P<0.05$ and ${ }^{\nabla \nabla} P<0.01$ versus group I.

and activating blood circulation may be a beneficial treatment for ALI. Dexamethasone was used as a positive control drug. It can also attenuate LPS-induced lung histopathological changes, decrease the wet/dry weight ratio and vascular leakage, and have anti-inflammatory mechanism.

The lung wet/dry weight ratio is an index reflecting the magnitude of pulmonary edema, resulting from increased alveolar capillary permeability and the accumulation of a large number of proteins in fluid [29]. In the current study, the lung wet/dry ratio of the herb I (AM/SM treatment after LPS administration) and herb II (AM/SM treatment before and after LPS administration) groups was lower than that of the LPS group, suggesting that AM/SM has protective therapeutic effects on LPS-induced ALI. As protein extravasation, resulting from widespread destruction of alveolar epithelium, is considered an indicator of vascular leakage [30], we measured the total protein concentration in BALF. We found that total protein concentrations in both Dex group and herb groups were remarkably reduced compared with the LPS group, while the therapeutic effect of dexamethasone was better than that of herb.

To further evaluate the effects of AM/SM on LPS-induced ALI, lung histological changes were detected. Microscopically, the lungs in the LPS group showed significant pathological changes, including pulmonary congestion, interstitial edema, alveolar wall thickening, and inflammatory cell infiltration. The magnitude of these changes decreased in the herb groups. These results suggest that AM/SM can improve pathological lung changes and attenuate pulmonary edema and vascular leakage, indicating that AM/SM has protective and therapeutic effects on LPS-induced ALI.

Proinflammatory cytokines, produced in the early phase of ALI, can amplify the inflammatory responses and result in pathological injuries in ALI $[31,32]$. TNF- $\alpha$ and IL-6 


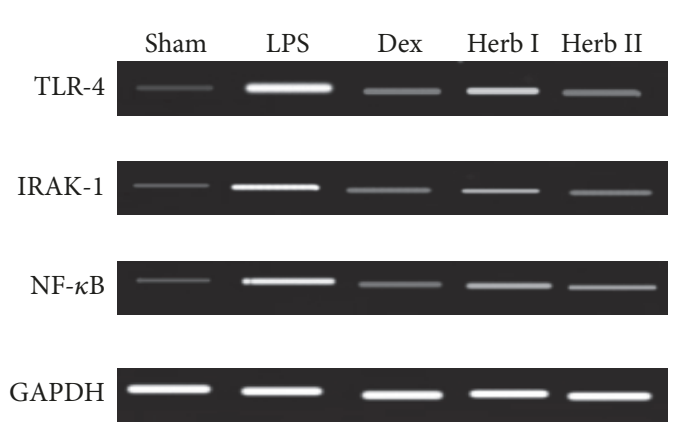

(a)

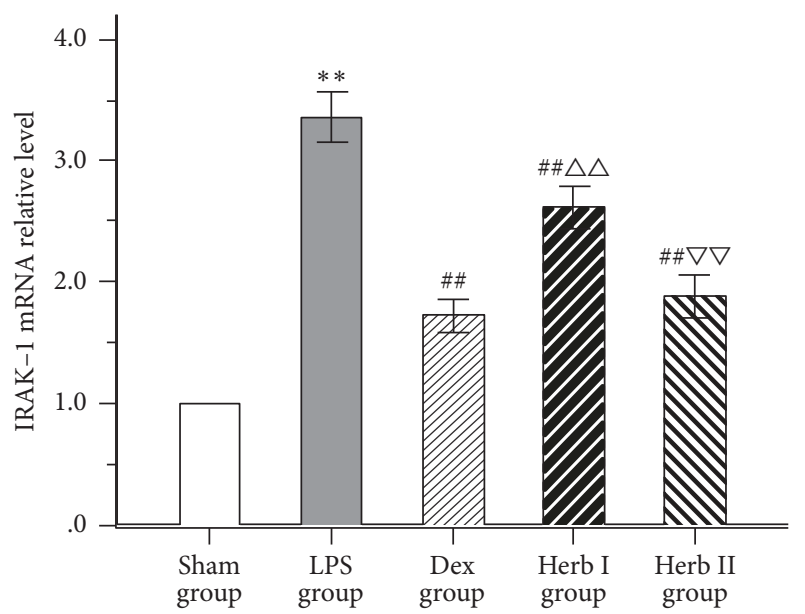

(c)

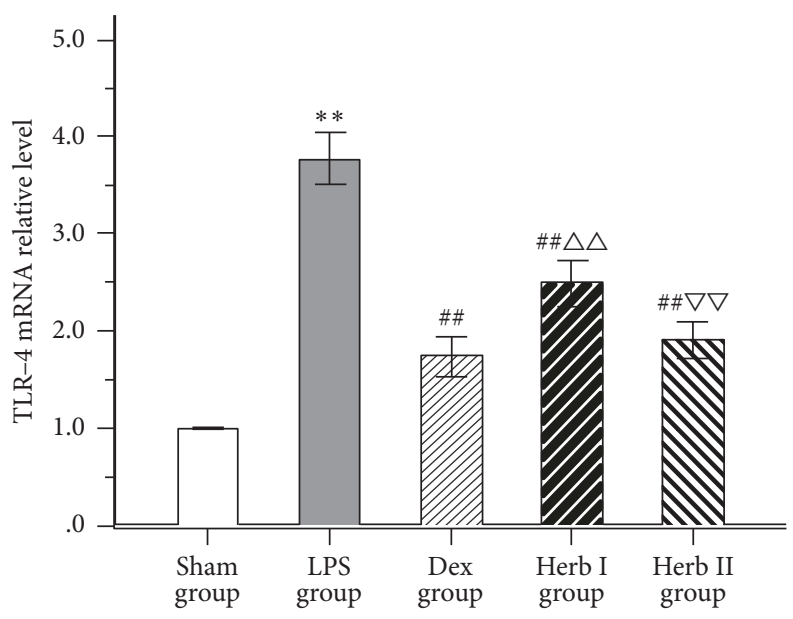

(b)

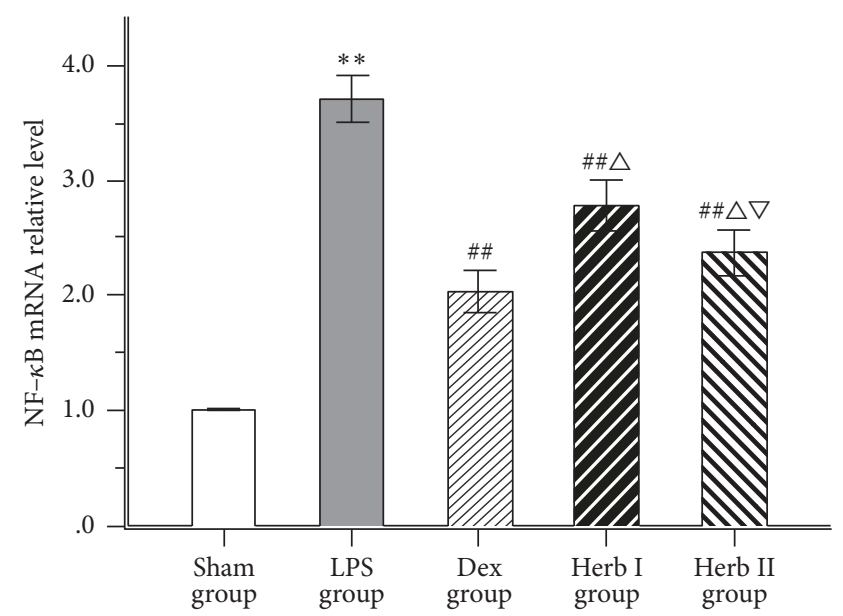

(d)

FIGURE 6: Effects of AM/SM on the TLR-4, IRAK-1, and NF- $\kappa$ B mRNA expression of lung tissues. (a) displays the pictures of mRNA abundance in the lung tissue. Levels of TLR-4 (b), IRAK-1 (c), and NF- $\kappa$ B/P65 (d) were standardized to GAPDH content. Values were expressed as mean $\pm \mathrm{SD}(n=10) .{ }^{* *} P<0.01$ versus sham group. ${ }^{\# \#} P<0.01$ versus LPS group. ${ }^{\triangle} P<0.05$ and ${ }^{\triangle \Delta} P<0.01$ versus Dex group. ${ }^{\nabla} P<0.05$ and ${ }^{\nabla \nabla} P<0.01$ versus group $\mathrm{I}$.

are characteristic early cytokines associated with the inflammatory process of ALI $[30,33]$. TNF- $\alpha$ plays an important role in lung tissue damage and dysfunction, and the level of TNF- $\alpha$ is associated with the degree of tissue damage [34]. As a cytokine with a primary and strong effect, TNF- $\alpha$ can damage vascular endothelial cells, inhibit the release of the alveolar surface-active substances, and increase alveolar capillary permeability and pulmonary edema [35]. Furthermore, TNF- $\alpha$ can induce the production of other inflammatory cytokines, including IL-6, which stimulates the migration and adherence of neutrophils to endothelial cells [36]. If the level of IL- 6 continues to increase in inflammatory diseases, complications and mortality are often accompanied [37]. In the current study, LPS challenge caused a significant increase in TNF- $\alpha$ and IL-6 levels in BALF, whereas dexamethasone and AM/SM significantly lowered the levels, with the effects in the herb II group and Dex group being stronger than those of the herb I group. These results suggest that the effects of AM/SM on this rat model of LPS-induced ALI were partly attributed to the inhibition of inflammatory factors. Dexamethasone can also attenuate the inflammatory reaction in the lung and herb group II was comparable to Dex group for the anti-inflammatory effect.

To explore the possible molecule mechanisms behind the protective and therapeutic effects of AM/SM on LPS-induced ALI, we examined the activity of TLR- $4 / N F-\kappa B$ signaling pathways in the lung. TLR- 4 is one of the most important signal-transducing receptors for structurally diverse microbial molecules (LPS) and can activate NF- $\kappa$ B to regulate the expression of inflammatory cytokines [12, 38]. Several lines of evidence suggest that upregulated expression of TLR4 in lung tissue is closely associated with high mortality and pathophysiology of ALI [34, 39]. Critical signaling pathways that are initiated after interaction of TLR- 4 involve IRAK-1 [40, 41]. Activation of IRAK-1 results in increased transcription of $\mathrm{NF}-\kappa \mathrm{B} / \mathrm{p} 65$, which is an important nuclear transcription factor and a regulator of many genes involved in ALI $[14,42]$. Previous studies have reported that NF- $\kappa \mathrm{B}$ 


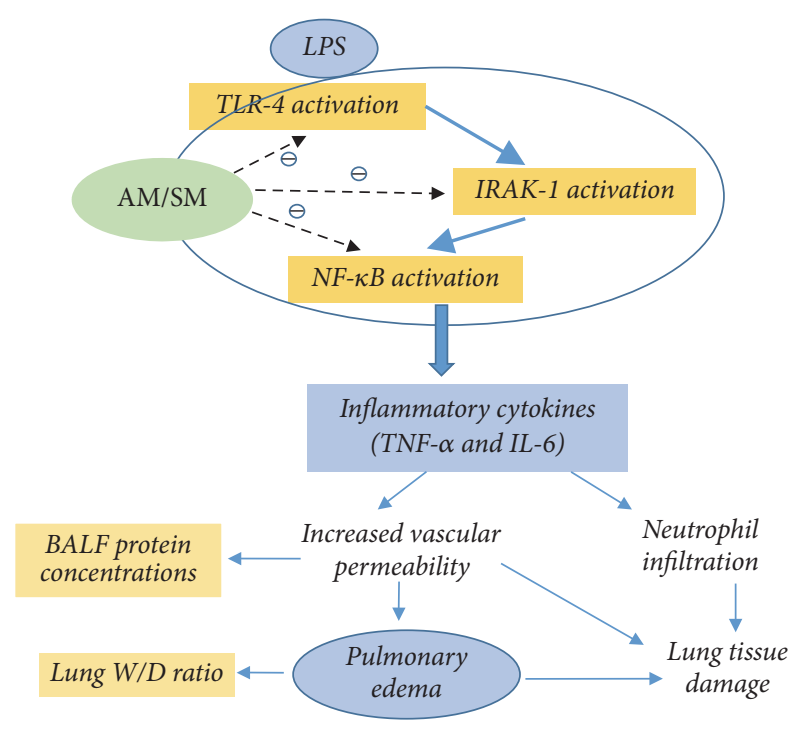

FIGURE 7: Schematic plot of the possible mechanisms behind the protective and therapeutic effects of AM/SM on LPS-induced ALI. AM/SM downregulated the TLR-4/IRAK-1/NF- $\kappa$ B signaling pathway; then inflammatory cytokines were decreased. Pulmonary edema and lung tissue damage were alleviated at last.

plays pivotal roles in immune and inflammatory responses through the regulation of the expression of several proteins, including proinflammatory cytokines, adhesion molecules, and chemokines $[43,44]$. A large body of research indicates that treatments focusing on regulating TLR-4/NF- $\kappa \mathrm{B}$ signaling might play a positive role on the resolution of lung injury [27, 30, 39, 45].

Some surface receptors (TLR-4), intracellular signaling molecules (IRAK-1), and nuclear activation factors (NF- $\kappa \mathrm{B}$ ) involved in LPS responses are functionally altered in ALI. In the current study, we used qPCR and immunohistochemistry to detect mRNA and protein expression of TLR-4, IRAK1 , and NF- $\kappa \mathrm{B} / \mathrm{p} 65$, as appropriate. With stimulation by LPS, TLR-4, IRAK-1, and NF- $\kappa$ B/p65 mRNA levels showed remarkable increases, as did protein levels (as evidenced by brown particles on imaging, which is reflective of a positive signal). Both herb groups showed decreased expression levels of these molecules compared with the LPS group, with decreased levels being most apparent in the herb II group. These results suggest that AM/SM showed protective effects in LPS-induced ALI rat through downregulation of the TLR$4 / \mathrm{IRAK}-1 / \mathrm{NF}-\kappa \mathrm{B}$ signaling pathway.

\section{Conclusions}

In this rat model of LPS-induced ALI, AM/SM administration improved lung histopathological changes, reduced lung wet/dry weight ratio, and decreased vascular leakage and inflammatory cytokine release. The effects of the prophylactic-therapeutic measure were observed to be the best. The mechanism behind AM/SM for the prevention and treatment of ALI may be associated with downregulation of the TLR-4/IRAK-1/NF- $\kappa \mathrm{B}$ signaling pathway (Figure 7 ).
The results of this study suggest that nourishing Qi and activating blood circulation show potential as an efficient therapeutic treatment for ALI in clinical application. Further comprehensive studies are required to examine other possible molecular mechanisms.

\section{Conflicts of Interest}

The authors declare no conflicts of interest.

\section{Acknowledgments}

This work was supported by the National Science Foundation of China (Grant no. 81303131).

\section{References}

[1] M. A. Matthay, L. B. Ware, and G. A. Zimmerman, "The acute respiratory distress syndrome," The Journal of Clinical Investigation, vol. 122, no. 8, pp. 2731-2740, 2012.

[2] A. P. Wheeler and G. R. Bernard, "Acute lung injury and the acute respiratory distress syndrome: a clinical review," The Lancet, vol. 369, no. 9572, pp. 1553-1564, 2007.

[3] A. Piper, Y. Song, N. D. Eves, and T. M. Maher, "Year in review 2013: acute lung injury, interstitial lung diseases, sleep and physiology," Respirology, vol. 19, no. 3, pp. 428-437, 2014.

[4] J. Phua, J. R. Badia, N. K. J. Adhikari et al., "Has mortality from acute respiratory distress syndrome decreased over time? A systematic review," American Journal of Respiratory and Critical Care Medicine, vol. 179, no. 3, pp. 220-227, 2009.

[5] G. D. Rubenfeld, E. Caldwell, E. Peabody et al., "Incidence and outcomes of acute lung injury," The New England Journal of Medicine, vol. 16, no. 353, pp. 1685-1693, 2005.

[6] V. A. K. Rathinam and K. A. Fitzgerald, "Immunology: lipopolysaccharide sensing on the inside," Nature, vol. 501, no. 7466, pp. 173-175, 2013.

[7] G. Matute-Bello, C. W. Frevert, and T. R. Martin, "Animal models of acute lung injury," American Journal of PhysiologyLung Cellular and Molecular Physiology, vol. 295, no. 3, pp. L379-L399, 2008.

[8] S. Akira, K. Takeda, and T. Kaisho, "Toll-like receptors: critical proteins linking innate and acquired immunity," Nature Immunology, vol. 2, no. 8, pp. 675-680, 2001.

[9] T. Kaisho and S. Akira, "Toll-like receptor function and signaling," The Journal of Allergy and Clinical Immunology, vol. 117, no. 5, pp. 979-987, 2006.

[10] H. Ohnishi, H. Tochio, Z. Kato et al., "Structural basis for the multiple interactions of the MyD88 TIR domain in TLR4 signaling," Proceedings of the National Acadamy of Sciences of the United States of America, vol. 106, no. 25, pp. 10260-10265, 2009.

[11] L. Ringwood and L. Li, "The involvement of the interleukin1 receptor-associated kinases (IRAKs) in cellular signaling networks controlling inflammation," Cytokine, vol. 42, no. 1, pp. $1-7,2008$.

[12] Y. Imai, K. Kuba, G. G. Neely et al., "Identification of oxidative stress and Toll-like receptor 4 signaling as a key pathway of acute lung injury," Cell, vol. 133, no. 2, pp. 235-249, 2008. 
[13] R. Yang, L. Yang, X. Shen et al., "Suppression of NF- $\kappa$ B pathway by crocetin contributes to attenuation of lipopolysaccharideinduced acute lung injury in mice," European Journal of Pharmacology, vol. 674, no. 2-3, pp. 391-396, 2012.

[14] X. Huang, J. Tang, H. Cai et al., "Anti-inflammatory effects of monoammonium glycyrrhizinate on lipopolysaccharideinduced acute lung injury in mice through regulating nuclear factor-kappa B signaling pathway," Evidence-Based Complementary and Alternative Medicine, vol. 2015, Article ID 272474, 8 pages, 2015.

[15] V. M. Bratkov, A. M. Shkondrov, P. K. Zdraveva, and I. N. Krasteva, "Flavonoids from the genus astragalus: phytochemistry and biological activity," Pharmacognosy Reviews, vol. 10, no. 19, pp. 11-32, 2016.

[16] J. Fu, Z. H. Wang, L. F. Huang et al., "Review of the botanical characteristics, phytochemistry, and pharmacology of Astragalus membranaceus (Huangqi)," Phytotherapy Research, vol. 28, no. 9, pp. 1275-1283, 2014.

[17] C.-Y. Su, Q.-L. Ming, K. Rahman, T. Han, and L.-P. Qin, "Salvia miltiorrhiza: traditional medicinal uses, chemistry, and pharmacology," Chinese Journal of Natural Medicines, vol. 13, no. 3, pp. 163-182, 2015.

[18] S. Gao, Z. Liu, H. Li, P. J. Little, P. Liu, and S. Xu, "Cardiovascular actions and therapeutic potential of tanshinone IIA," Atherosclerosis, vol. 220, no. 1, pp. 3-10, 2012.

[19] Y. Zhou, W. Li, L. Xu, and L. Chen, "In Salvia miltiorrhiza, phenolic acids possess protective properties against amyloid $\beta$-induced cytotoxicity, and tanshinones act as acetylcholinesterase inhibitors," Environmental Toxicology and Pharmacology, vol. 31, no. 3, pp. 443-452, 2011.

[20] X. Chen, J. Guo, J. Bao, J. Lu, and Y. Wang, “The anticancer properties of salvia miltiorrhiza Bunge (Danshen): a systematic review," Medicinal Research Reviews, vol. 34, no. 4, pp. 768-794, 2014.

[21] X.-H. Ma, Y. Ma, J.-F. Tang et al., "The biosynthetic pathways of tanshinones and phenolic acids in salvia miltiorrhiza," Molecules, vol. 20, no. 9, pp. 16235-16254, 2015.

[22] M. Li, M.-H. Yang, Y.-Q. Dou, and Y. Liu, "Clinical research on supplementing Qi and activating blood circulation herbs on lung protection in acute lung injury ventilation patients," Zhong Yao Cai, vol. 37, no. 11, pp. 2113-2116, 2014.

[23] M. A. Matthay and R. L. Zemans, "The acute respiratory distress syndrome: pathogenesis and treatment," Annual Review of Pathology: Mechanisms of Disease, vol. 6, pp. 147-163, 2011.

[24] H.-M. Liu and Y.-N. Guo, "Effect of captopril on serum TNFalpha level in acute lung injury rats induced by HCL," Asian Pacific Journal of Tropical Medicine, vol. 7, no. 11, pp. 905-908, 2014.

[25] Y. Butt, A. Kurdowska, and T. C. Allen, "Acute lung injury: a clinical and molecular review," Archives of Pathology \& Laboratory Medicine, vol. 140, no. 4, pp. 345-350, 2016.

[26] H. Chen, C. Bai, and X. Wang, "The value of the lipopolysaccharide-induced acute lung injury model in respiratory medicine," Expert Review of Respiratory Medicine, vol. 4, no. 6, pp. 773-783, 2010.

[27] X. Zhang, C.-Y. Sun, Y.-B. Zhang et al., "Kegan Liyan oral liquid ameliorates lipopolysaccharide-induced acute lung injury through inhibition of TLR4-mediated NF- $\kappa$ B signaling pathway and MMP-9 expression," Journal of Ethnopharmacology, vol. 186, pp. 91-102, 2016.

[28] W. Jiang, F. Luo, Q. Lu et al., "The protective effect of Trillin LPSinduced acute lung injury by the regulations of inflammation and oxidative state," Chemico-Biological Interactions, pp. 127134, 2015.

[29] M. Rojas, C. R. Woods, A. L. Mora, J. Xu, and K. L. Brigham, "Endotoxin-induced lung injury in mice: structural, functional, and biochemical responses," American Journal of PhysiologyLung Cellular and Molecular Physiology, vol. 288, no. 2, pp. L333-L341, 2005.

[30] G. Feng, B. Sun, and T.-Z. Li, "Daidzein attenuates lipopolysaccharide-induced acute lung injury via toll-like receptor 4/NF-kappaB pathway," International Immunopharmacology, vol. 26, no. 2, pp. 392-400, 2015.

[31] R. B. Goodman, J. Pugin, J. S. Lee, and M. A. Matthay, "Cytokine-mediated inflammation in acute lung injury," Cytokine \& Growth Factor Reviews, vol. 14, no. 6, pp. 523-535, 2003.

[32] M. Bhatia and S. Moochhala, "Role of inflammatory mediators in the pathophysiology of acute respiratory distress syndrome," The Journal of Pathology, vol. 202, no. 2, pp. 145-156, 2004.

[33] W. Jing, M. Chunhua, and W. Shumin, "Effects of acteoside on lipopolysaccharide-induced inflammation in acute lung injury via regulation of NF- $\kappa$ B pathway in vivo and in vitro," Toxicology and Applied Pharmacology, vol. 285, no. 2, pp. 128-135, 2015.

[34] C. Xu, G. Chen, W. Yang et al., "Hyaluronan ameliorates LPSinduced acute lung injury in mice via Toll-like receptor (TLR) 4dependent signaling pathways," International Immunopharmacology, vol. 28, no. 2, pp. 1050-1058, 2015.

[35] S. Mukhopadhyay, J. R. Hoidal, and T. K. Mukherjee, "Role of TNF $\alpha$ in pulmonary pathophysiology," Respiratory Research, vol. 7, article 125, 2006.

[36] X. Liang, R. S. Wang, F. Wang et al., "Sodium butyrate protects against severe burn-induced remote acute lung injury in rats," PLoS ONE, vol. 8, no. 7, Article ID e68786, 2013.

[37] G. Mastorakos and I. Ilias, "Interleukin-6: A cytokine and/or a major modulator of the response to somatic stress," Annals of the New York Academy of Sciences, vol. 1088, pp. 373-381, 2006.

[38] Y. Lu, W. Yeh, and P. S. Ohashi, "LPS/TLR4 signal transduction pathway," Cytokine, vol. 42, no. 2, pp. 145-151, 2008.

[39] G. Feng, Z.-Y. Jiang, B. Sun, J. Fu, and T.-Z. Li, "Fisetin alleviates lipopolysaccharide-induced acute lung injury via TLR4mediated NF- $\kappa$ B signaling pathway in rats," Inflammation, vol. 39, no. 1, pp. 148-157, 2016.

[40] S. Janssens and R. Beyaert, "Functional diversity and regulation of different interleukin-1 receptor-associated kinase (IRAK) family members," Molecular Cell, vol. 11, no. 2, pp. 293-302, 2003.

[41] Y. Li, E. Annette Howell, A. S. Lagoo et al., "Differential gene expression of interleukin-1 receptor associated kinase-1 and interleukin-1 receptor associated kinase- $m$ in peripheral blood mononuclear cells of young and aged rats following preconditioning with endotoxin," Shock, vol. 31, no. 1, pp. 5563, 2009.

[42] T. Zhu, D. X. Wang, W. Zhang et al., "Andrographolide protects against LPS-induced acute lung injury by inactivation of NF«B," PLoS ONE, vol. 8, no. 2, Article ID e56407, 2013.

[43] B. Hoesel and J. A. Schmid, "The complexity of NF- $\kappa$ B signaling in inflammation and cancer," Molecular Cancer, vol. 12, no. 1, article 86, 2013.

[44] H. Wang, L. Xu, and J. Zhao, "Regulatory mechanism of pyrrolidine dithiocarbamate is mediated by nuclear factor- $\kappa \mathrm{B}$ and inhibits neutrophil accumulation in ARDS mice," Experimental and Therapeutic Medicine, vol. 8, no. 2, pp. 614-622, 2014. 
[45] Z. Tianzhu and W. Shumin, "Esculin inhibits the inflammation of LPS-induced acute lung injury in mice via regulation of TLR/NF- $\kappa$ B pathways," Inflammation, vol. 38, pp. 1529-1536, 2015. 


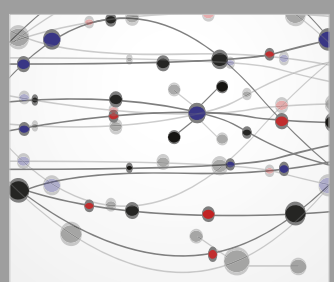

The Scientific World Journal
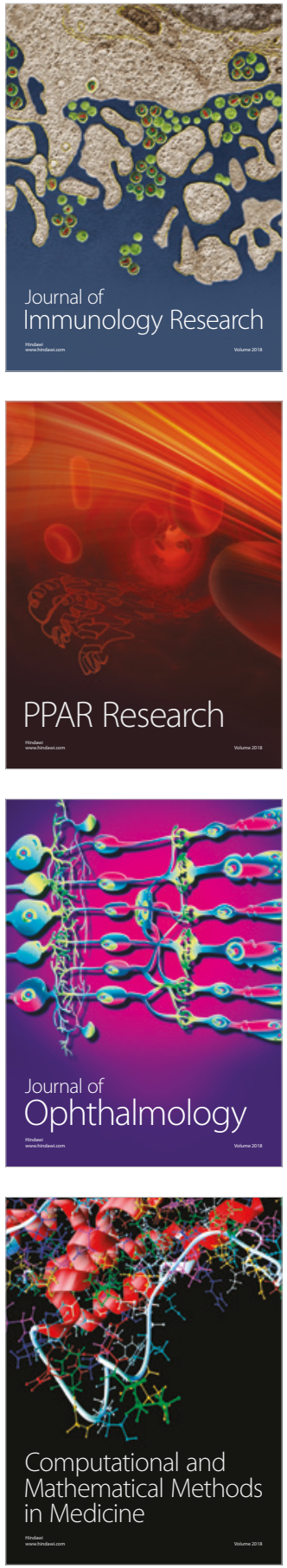

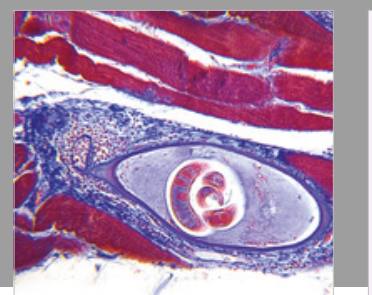

Gastroenterology Research and Practice

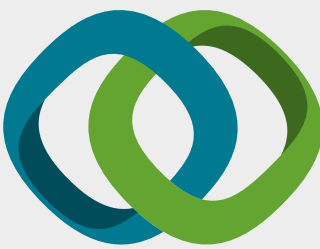

\section{Hindawi}

Submit your manuscripts at

www.hindawi.com
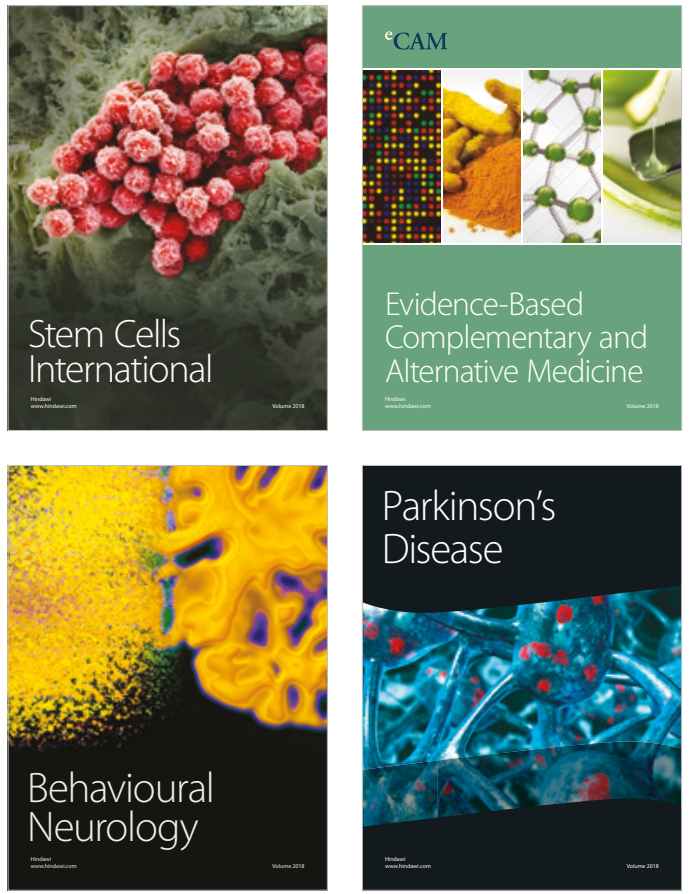

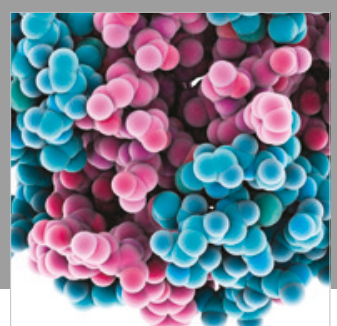

ournal of

Diabetes Research

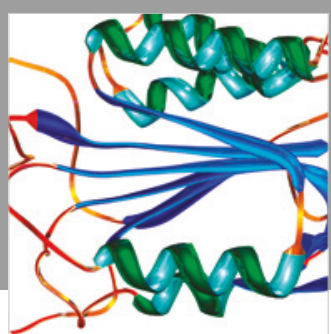

Disease Markers
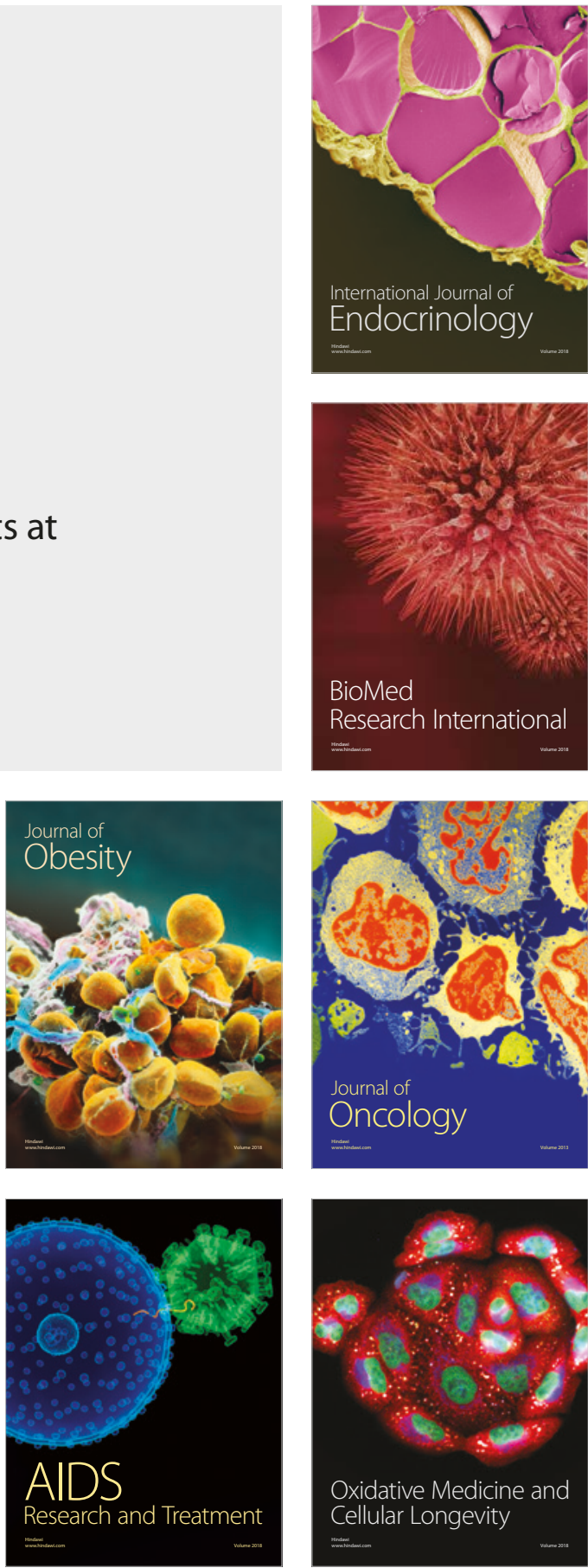